\title{
RECONSTRUCTION OF UNDERWATER IMAGE BY BISPECTRUM
}

\author{
Z.Y. Wen, D. Fraser, A. Lambert.
}

School of ITEE

The University of New South Wales

Australian Defence Force Academy, Canberra
H.D. $\mathrm{Li}$

\author{
RSISE, Australian National University \\ VISTA, National ICT Australia \\ Canberra, Australia
}

\begin{abstract}
Reconstruction of an underwater object from a sequence of images distorted by moving water waves is a challenging task. A new approach is presented in this paper. We make use of the bispectrum technique to analyze the raw image sequences and recover the phase information of the true object. We test our approach on both simulated and real-world data, separately. Results show that our algorithm is very promising. Such technique has wide applications to areas such as ocean study and submarine observation.
\end{abstract}

Index Terms - bispectrum, water wave, image, reconstruction, distortion, refraction.

\section{INTRODUCTION}

Assume that we look through the moving water surface and observe an underwater object (for example, a coral reef), we would notice that the object is distorted due to the effect of light refraction and motion blur. Such phenomena can be recorded by a stationary video camera above the water. Each image in the video stream may be degraded and blurred. Figure 1 shows a sample sequence of distorted images.

How to recover the target image from the video stream (distorted images) is a very challenging and a cross-disciplinary task, which involves physics, optics, computer vision and image processing. Such technology has wide applications such as underwater surveillance and submarine observation.

In past decades, researchers from different areas have addressed the problem of recovering underwater image $[1,2$, 3]. Some focused on reconstructing the surface of the water, some tried statistical theory to recover the target, some studied light refraction, and some applied image processing techniques. One simple method is the average-based method, which temporally averages the data stream [1]. Another method is to locate the minimum distorted regions and form the final image from these regions [2,3]. Both methods work well in many situations. The latter method works better under severe disturbed conditions.

This work is supported by University College, UNSW@ADFA. NICTA is funded through the Australian Government, in part through the ARC.

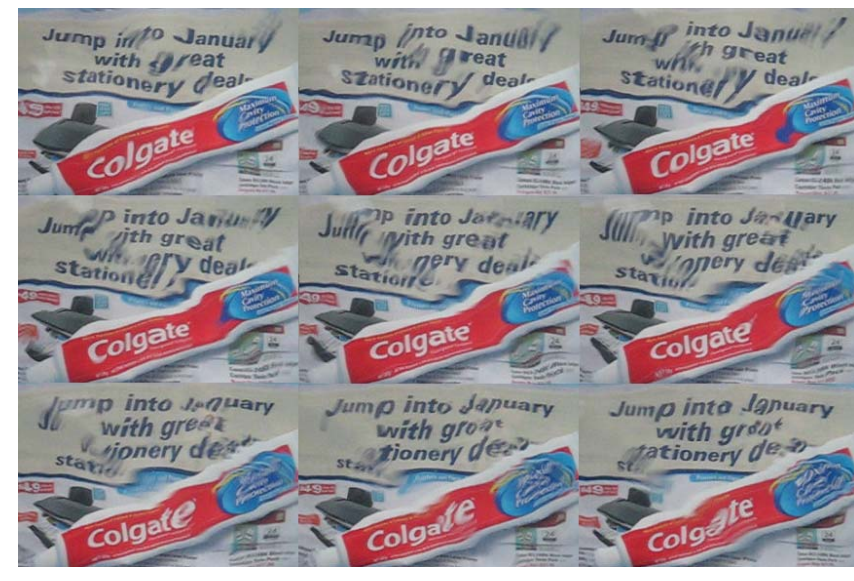

Fig. 1. A sample sequence of distorted images.

In this paper, an original approach to obtaining the true image from the sequence of the distorted images is proposed. We estimate the Fourier phase of the target image by analyzing the averaged bispectrum of the image ensemble. Results of our experiments show that our algorithm is promising.

\section{PROBLEM STATEMENT}

Before constructing an algorithm, it is worth introducing the physical background of our problem. Figure 2 demonstrates the refraction law. Assume that light passes straight up from the planar ground under the water, there will be no refraction if the water surface is still. Under this condition, the observer at point a will see the object $\mathbf{O}$. However, when the water waves exist, the normal to the water surface $\mathbf{N}$ is tilted by an angle. This results in that the observer at point $\mathbf{b}$ will see $\mathbf{O}^{\prime}$ instead of $\mathbf{O}$. According to Snell's law, the refractive index of a material is calculated by the angle $\mathbf{c}$ and $\mathbf{d}$. Here, the index of refraction of water is 1.33 .

When recording a particular object under the moving water surface over time, we will see that the object is distorted and moves around its correct position. These movements are Gaussian distributed with its center being around the cor- 


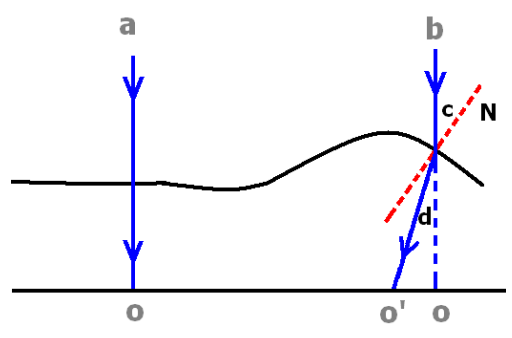

Fig. 2. A simple illustration of the refraction law.

rect position in accordance with Cox-Munk Law[4]. The law points out that, given a large surface area and stationary waves, the distribution of the normals of the water surface is approximately Gaussian. Efros et. al. confirmed this statement by experiments[2]. Therefore the properties of Gaussian random process can be utilized when processing the images.

The most common approach to such a problem is the averagebased method. The average-based method is to compute the temporal average over the image ensemble[1]. It works well under comparatively smooth conditions. However, it fails when the target is too fine and has a lot of details. Recently, several researchers proposed to form the target image by locating and combining the minimum distorted regions over the sequence of the raw images[2, 3, 5]. The result of such method is much sharper than that of the average-based method.

In this paper, we consider the task as a phase recovery problem of images captured under disturbed conditions, so that the bispectrum technique can be used to recover the correct phase of the target. Bispectrum techniques are primarily used in astronomical imaging to reconstruct the target affected by atmospheric turbulence[6]. To apply the technique to our problem, a sequence of raw images are required. We applied such a technique in our problem and achieved promising results.

\section{BISPECTRUM TECHNIQUE}

In this section, we give a brief review on the main technique of our algorithm.

Assume that $I(u, v)$ is the Fourier transform of a 2-D signal $i(x, y)$ (for example, an image), which is also expressed as the magnitude-and-phase form:

$$
I(u, v)=|I(u, v)| \exp \left\{j \phi_{i}(u, v)\right\}
$$

From $\mathrm{Eq}(1)$, a signal can be recovered given that the magnitude and the phase in the Fourier domain are known. In this paper, the bispectrum technique is used to recover the signal phase.

The bispectrum has two main properties: (1) the phase information of a signal can be preserved and (2) the bispectrum of a Gaussian signal is zero. This enables us to recover the correct phase of a signal using the bispectrum technique.

The bispectrum is the Fourier form of the triple correlation of a signal. The bispectrum of a 2-D signal is given by

$$
\begin{aligned}
& B\left(u_{1}, u_{2} ; v_{1}, v_{2}\right) \\
& =I\left(u_{1}, u_{2}\right) I\left(v_{1}, v_{2}\right) I^{*}\left(u_{1}+v_{1}, u_{2}+v_{2}\right) \\
& =I\left(u_{1}, u_{2}\right) I\left(v_{1}, v_{2}\right) I\left(-\left(u_{1}+v_{1}\right),-\left(u_{2}+v_{2}\right)\right)
\end{aligned}
$$

where $B\left(u_{1}, u_{2} ; v_{1}, v_{2}\right)$ denotes the bispectrum, and $*$ indicates the complex conjugate. Note that the bispectrum of a 2-D signal is four dimensional.

Since the normal of the water surface is a Gaussian distribution, the phase distortion of a submerged object is also considered as Gaussian distributed. This allows us to use the properties of Gaussian random process and overcome the phase corruption by averaging the bispectrum of the ensemble of the raw images [6]. So that we build the relationship between the object phase spectrum $\phi_{O}(u, v)$ and the phase of the mean bispectrum $\phi_{B}\left(u_{1}, u_{2} ; v_{1}, v_{2}\right)$ by

$$
\begin{aligned}
& \phi_{o}\left(u_{1}+v_{1}, u_{2}+v_{2}\right) \\
& =\phi_{o}\left(u_{1}, u_{2}\right)+\phi_{o}\left(v_{1}, v_{2}\right)-\phi_{B}\left(u_{1}, u_{2} ; v_{1}, v_{2}\right) .
\end{aligned}
$$

From Eq.(3), one can estimate the phases at higher frequencies using the phases at lower frequencies and the mean bispectrum phases given that the phases at $( \pm 1,0)$ and $(0, \pm 1)$ are known (the phase at $(0,0)$ is zero) $[7,8,9]$.

\section{ALGORITHM}

From the above analysis, we now construct our algorithm.

The first step is to divide the raw images into smaller size patches and locate and discard the most distorted patches. Such a method is similar to the lucky imaging technique in astronomical imaging[10, 11], which aims to select the good images to recover the target image. In the problem, Efros et. al. show that one particular spot under the water surface suffers different distortion in temporal space[2]: the local image patch is more distorted under higher energy water wave surface and less while the water surface is nearly flat. Here we employ the image quality index proposed in [12] as the selection criterion.

$$
Q=\frac{4 \sigma_{x y} \bar{x} \bar{y}}{\left(\sigma_{x}^{2}+\sigma_{y}^{2}\right)\left[\bar{x}^{2}+\bar{y}^{2}\right]}
$$

where $x$ is the clean image and $y$ is the test image, $\bar{x}$ and $\bar{x}^{2}$, and $\bar{y}$ and $\bar{y}^{2}$ are the expectation and the variance of $x$ and $y$, respectively, $\sigma_{x y}$ is the covariance of $x$ and $y$. Because the clean image is unknown and is what we are trying to find, we substitute the mean of the raw image ensemble for $x$.

We then estimate the Fourier phase and the Fourier magnitude of each patch of the target image after discarding the 
most distorted patches. The Fourier magnitude is estimated by the averaged power spectrum of the image ensemble in our experiment.

The phase of the object in the Fourier domain is recovered using Eq.(3). Here, the recursive algorithm is employed [7]. Since the exact values of the phases at $( \pm 1,0)$ and $(0, \pm 1)$ are unknown, we use the mean image of the image ensemble here again: $\phi_{o}( \pm 1,0)=\phi_{\text {mean }}( \pm 1,0)$ and $\phi_{o}(0, \pm 1)=$ $\phi_{\text {mean }}(0, \pm 1)$.

The final step is to reconstruct each patch of the target with the Fourier magnitude and phase obtained in the previous step, and to combine the patches to their right positions to form the final image.

\section{EXPERIMENTS}

We test our algorithm on both simulated and real-world data. Results show that our algorithm is promising.

\subsection{Simulation}

\section{simulation 123 wave silface}

(a) One of the input images

\section{simulation 123} wave surface

(c) Result by our algorithm lengths, they can be expressed by the wave number $u_{i}$ and $v_{i}$, the angular frequency $w_{i}$ and the amplitude $a_{i}$ as follows:

$$
\left\{\begin{array}{l}
p=-\sum a_{i} u_{i} \cos \left(u_{i} x+v_{i} y-w_{i} t\right) \\
q=-\sum a_{i} v_{i} \cos \left(u_{i} x+v_{i} y-w_{i} t\right)
\end{array}\right.
$$

By adjusting $h, u_{i}$ and $v_{i}$, we can obtain different types of waves and therefore simulate smooth and deep water waves.

We generate a sequence of 100 gray images of size $256 \times 96$. The size of each image tile used in our experiments is $32 \times 32$. Figure 3(a) is a sample distorted image simulated by the model, (b) the averaged image of the data sequence over time, (c) is the output of our algorithm, and (d) the true image. Note that the image estimated by our algorithm is much sharper than that by the average-based method, and more precisely represents the true image.

\subsection{Real-world Results}

In an experiment with a real water surface, the natural data is captured by a video camera fixed above the water surface with the object laid on the planar ground under the water. The moving water surface is motivated by natural factors (e.g., wind produced by a fan and an object dropped into the water). The water is clear and no account has been taken of the attenuation of the image by a turbid media.

Figure 1 shows a sample sequence of the real-world data. The image size is $288 \times 192$. In total 120 images are used and the size of image tiles is also $32 \times 32$. The comparison of reconstruction results by different methods is demonstrated in Figure 4. The result of our algorithm is promising, and the fine details of the object can be clearly seen.

\section{CONCLUSION}

We propose an original idea to reconstruct a submerged object distorted by moving water surface. We assume the normals of the water surface are Gaussian distributed. The bispectrum technique is employed to recover the phase of the true object. Although experiments show that our approach is promising, there exist some limits. One limit is that our algorithm needs a large computer memory and heavy computation because that the bispectrum of an image is four dimensional. Another limit is the recursive phase recovery method with only a subset of the phase information of the averaged bispectrum being used. This may reduce the resolution of the output. To overcome such limits is the next step in our research.

\section{REFERENCES}

[1] R Shefer, M. Malhi, and A. Shenhar, "Waves distortion correction using cross correlation," http://visl.technion.ac.il/projects/2000maor/, 2001. perposed patterns of water waves with different speeds and 


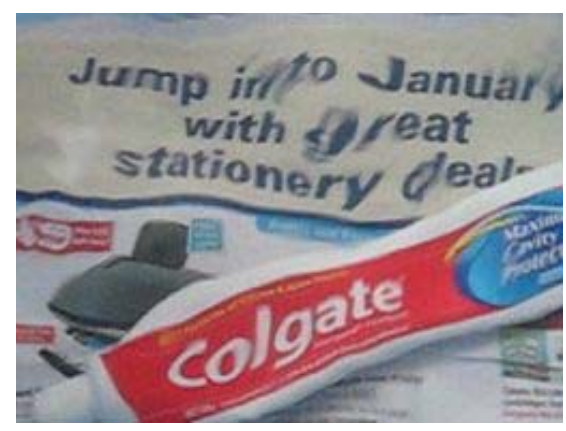

(a) One of the input images.

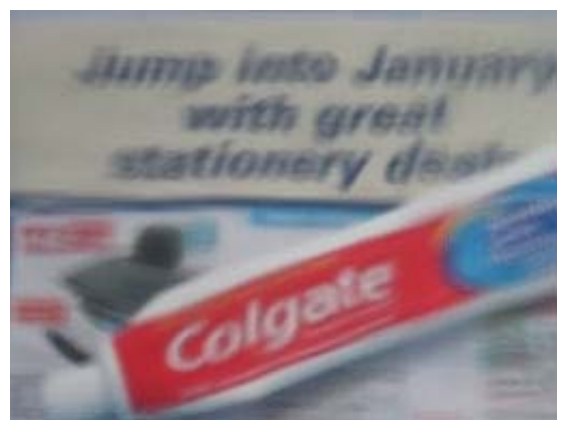

(c) Result by the lucky region method.

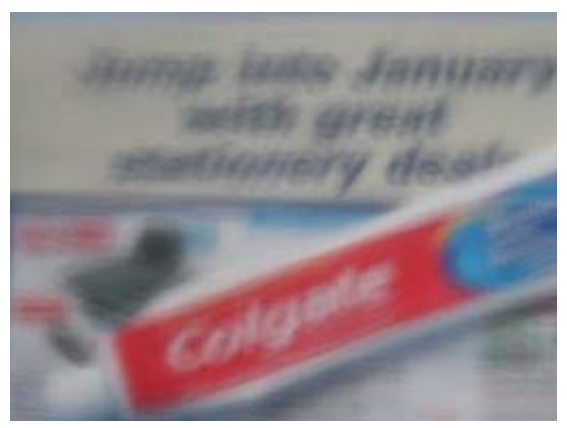

(b) Result by the average-based method.

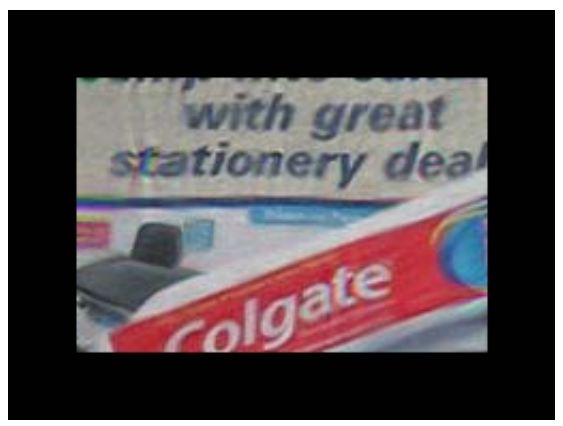

(d) Result by our method.

Fig. 4. Comparison of seeing-through-water reconstruction results of different methods.

[2] A. Efros, V. Isler, J. Shi, and M. Visontai, "Seeing through water," NIPS, 2005.

[3] A. Donate and E. Ribeiro, "Improved reconstructin of images distorted by water waves," International conference on computer vision theory and applications, 2006.

[4] C. Cox and W. Munk, "Slopes of the sea surface deduced from photographs of sun glitter," Scripps Inst. of Oceanogr, vol. 6, pp. 401-479, 1956.

[5] A. Donate, G. Dahme, and E. Ribeiro, "Classification of textures distorted by water waves," ICPR, 2006.

[6] M. Roggemann and B. Welch, Imaging Through turbulence, CRC Press LLC, 1996.

[7] A. Lohmann and B. Wirnitzer, "Triple correlations," Proc. of IEEE, vol. 72, pp. 889-901, July 1984.

[8] C. Matson, "Weighted-least-squares phase reconstruction from the bispectrum," J.Opt.Soc.Am.A, vol. 8, pp. 1905-1913, 1991.

[9] H. Bartle, A. Lohmann, and B. Wirnitzer, "Phase and amplitude recovery from bispectra," J.Opt.Soc.Am, vol. 23, pp. 3121-3129, Sept. 1984.
[10] D. Fried, "Probability of getting a lucky short-exposure image through turbulence," J.Opt.Soc.Am.A., vol. 68, pp. 1651-1658, 1978.

[11] S. Weddell and R. Webb, "Data preprocessing on sequential data for improved astronomical imaging," Image and Vision Computing, Nov 2005.

[12] Z. Wang and A. Bovik, "A universal image quality index," IEEE Signal Processing Letters, vol. 9, pp. 81-84, 2002.

[13] H. Murase, "Surface shape reconstruction of a nonrigid transparent object using refraction and motion," IEEE Transaction on Pattern Analysis and Machine Intelligence, Oct. 1992. 